\title{
Entomologische Ergebnisse einer Reise nach Oberitalien und Südtirol (1910).
}

(Neuroptera, Odonata, Orthoptera, Lepidoptera, Diptera, Hymenoptera, Rhynchota).

Von Willy Ramme, Charlottenburg.

Mit Tafel II.

Die von Mitte Mai bis Anfang August 1910 bereisten Gebiete Gardasee und unteres Eisacktal - bieten in faunistischer Beziehung ein besonderes Interesse dar, da sich hier die mitteleuropäische (im engern Sinne) und die mediterrane Fauna berühren. Ich habe daher auch nicht gezögert, alle gesammelten und beobachteten Arten aufzuführen; gerade in solchen Grenzgebieten wird es von Wichtigkeit sein, eine genaue Kenntnis der Fauna zu gewinnen, da wir nur so in der Lage sind, die Bewegung derselben, d. h. ein Vordringen bezw. Zurückweichen einzelner Arten zu erkennen, abgesehen davon, daß wir über die Verbreitung vieler Arten vorläufig überhaupt nur ungenau orientiert sind. Zudem dürfte es ganz allgemein jedem, der zu entomologischen Studienzwecken ein Gebiet bereisen will, willkommen sein, wenn möglichst viele und genaue Sammelberichte aus demselben vorliegen.

Soweit biologische Beobachtungen gemacht werden konnten, sind dieselben angeführt; ferner finden sich Beschreibungen einiger bemerkenswerterer, wenn auch nicht neu zu benennender Abweichungen; das bisher unbekannte of von Hoplismenus pica Wesm. (Ichneum.) wurde bei Klausen aufgefunden.

Die Reise begann ich am 19. Mai und durchstreifte bis zum 16. Juni das Gardaseegebiet; hauptsächlich sammelte ich bei Gardone (am Südwestufer), wo ich mich vom 21. Mai bis 14. Juni aufhielt; für die andern von mir besuchten Orte (Sirmione, Salò, Toscolano-Gaïno, Malcésine, Torbole, Riva) ist das Datum jedesmal angegeben. Der zweite Teil der Reise galt dem unteren Eisacktal; Standquartier war während der ganzen Zeit Klausen (18. Juni bis 3. August). Von einer im Juli 1905 nach dem Gardasee und nach Venedig unternommenen Reise sind einige wenige Notizen beigefügt.

In beiden Gebieten wurden fast ausschließlich die tieferen Lagen (bis zu etwa $600 \mathrm{~m}$ ) durchforscht; in Gardone kommt also hauptsächlich die Olivenregion und die sich anschließende Region der Eichen (Büsche 
und kleinere Bäume) in Betracht. Nadelwald ist, abgesehen von einigen Cypressengruppen, nicht vorhanden, da leider - wie überall in Italien - allmählich die Wälder heruntergeschlagen worden sind, ohne daß für neue Aufforstung Sorge getragen wurde. Das Seeufer ist meist kahl und steinig und daher unergiebig; auch an den von Schilf etc. bewachsenen Partien, wie z. B. bei Sirmione, war die Ausbeute nur gering. Überhaupt hat mich das Gardaseegebiet in entomologischer Hinsicht etwas enttäuschí, zumal da ich es diesmal in der günstigsten Jahreszeit besuchte ; immerhin war es doch noch recht ergiebig, wenn es auch in dieser Beziehung keinen Vergleich mit der Klausener Gegend aushält, die ja schon seit langem als ein Dorado für den Entomologen bekannt ist.

Es wurden beim Sammeln alle Insektenordnungen berücksichtigt; die Ausbeute betrug insgesamt etwa 750 Arten mit ca. 2500 Exemplaren. In dem vorliegenden Bericht sind zunächst die Ordnungen der Neuropteren (6 Arten), Odonaten (5 Art.), Orthopteren (26 Art.), Lepidopteren (165 Art.), Dipteren (80 Art.), Hymenopteren (87 Art.), und Rhynchoten (71 Arten) behandelt; die Coleopteren (325 Art.) und einige kleinere Ordnungen sollen ev. später folgen. Von den Coleopteren sei vorläufig nur eine Anomalie von Blaps mucronata Latr. mit zweiteiligem linken Fühler erwähnt. Taf. II, Fig. 1.

Die Bestimmungen dürften einwandfrei sein, da sie bei Ordnungen resp. Familien, die dem Verf. ferner lagen, von genauen Kennern derselben vorgenommen oder in zweifelhaften Fällen nachgeprüft wurden. Ich möchte nicht versäumen, den betr. Herren für ihre Bemühungen meinen verbindlichsten Dank auszusprechen; es sind dies die Herren Sanitätsrat Dr. Bastelberger-Würzburg (Geom.), H. B is c h of f-Berlin (Chrys.), Dr. Enslin-Fürth (Tenthr.), Prof. Dr. Krieger-Leipzig (Ichneum.), B. Lichtwardt-Charlottenburg und R. St ob be-Berlin (Dipt.), F. Schumacher-Kagel (Rhynch.), H. Stit z-Berlin (Formic.), E m b rik Strand-Berlin (Apid.).

\section{Neuroptera.}

1. Raphidia affinis Schneid. Klausen, häufig.

2. Myrmeleo formicalynx L. Klausen (groß, Flügelsp. $8 \mathrm{~cm}$ ).

3. - tetragrammicus F. Klausen, Atzwang, Ende Juli.

4. Megistopus flavicornis Rossi. 1 Exemplar am Mte. Castello bei Gaïno (nahe Gardone), 10. 6. Es dürfte dies der westlichste bisher beobachtete Punkt des Vorkommens dieser eigentlich südosteuropäischen Art sein.

5. Ascalaphus coccajus W. V. Gardone, 21. 5. 1 Exemplar.

6. Osmylus maculatus F. Klausen (Thinneschlucht).

\section{Odonata.}

1. Libeliula fulva L. $1 \sigma$, Gardone.

2. - depressa Müll. Gardone, Klausen. 
3. Gomphus forcipatus L. Malcésine, häufig am Seeufer.

4. Cordulegaster spec. An einem Teich zwischen Klausen und Villnöß war ein $C$. ungemein häufig; leider ging das einzige erbeutete Exemplar verloren, sodaß die Art nicht festgestellt werden konnte.

5. Calopteryx virgo L. Gardone, Klausen.

\section{Orthoptera.}

\section{Forficulidae.}

1. Labia minor L. Gardone, 20. 6. 1 Expl. kam ans Licht.

2. Forficula auricularia L. Überall unter Steinen gemein.

\section{Blattidae.}

3. Blatta orientalis L. In Häusern.

4. Ectobia lapponica L. Häufig; aus Salò ein Exempl. der f. pallida Stephens. Ist bisher teils als Aberration, teils als Varietät in Serbien (Brunner, Prodromus der eur. Orth. 1882), Belgien und Spanien (Burr, Synopsis of the Orthoptera of Western Europe, London 1910) beobachtet worden. Das betr. Exemplar aus Salò ist im Vergleich zu lapponica auffallend groß; es stellt in der Färbung wohl den extremsten Typus der Form dar, sodaß ich es zunächst für eine E. perspicillaris Herbst hielt und nur durch den Habitus auf lapponica geführt wurde. Um ganz sicher zu gehen, sandte ich das Tier an die Herren R. Shelford-Oxford und Dr. Malcolm Burr-Dover, die sich meiner Ansicht anschlossen; im Oxford-Museum befinden sich zwei ähnliche Stücke aus Österreich. Beiden Herren an dieser Stelle meinen verbindlichsten Dank für ihre Bemühungen! - Im folgenden die Beschreibung des Stückes:

Stirn orange, Pronotum in der Mttte rötlich-gelb mit abgesetztem helleren Rand. Thorax und Abdomen auf der Ventralseite glänzend schwarz, ebenso auf der Dorsalseite, hier jedoch am distalen Ende aufgehellt. Cerci und Subgenitalplatte ge 1b, Beine v o 11ständig gelb. Vfl. glänzend gelblich (auch die vena mediastina gelb), ohne jede Zeichnung. Nachstehend die Größenverhältnisse (die eingeklammerten Zahlen für typ. Lapponica).

$\begin{array}{lrll}\text { Pronotum } & 3,6 \mathrm{~mm} \text { br. } & (2,6) \\ & 2,1 \mathrm{~mm} \text { lg. } & (1,8) \\ \text { Fühler } & 12 \mathrm{~mm} & (9) \\ \text { Vorderflügel } & 12 \mathrm{~mm} & (8)\end{array}$

Ich möchte hier die auch von Shelford geäußerte Ansicht vertreten, daß perspicillaris und lapponica möglicherweise artlich garnicht von einander zu trennen sind, zumal man beide zu- 
weilen miteinander in copula findet; obige Aberration ist vielleicht ein Kreuzungsprodukt beider Arten.

5. Ectobia perspicillaris Herbst. (livida Fabr.) Gardone, in typischen Exemplaren.

\section{Mantidae.}

6. Mantis religiosa L. Ich fand nur Eipakete; obwohl $M$. am Gardasee wie auch in Südtirol sehr häufig ist, gelang es mir später trotz eifrigen Suchens nicht, Larven zu finden. Die Imagines treten erst Ende August auf. Erstere sollen sehr versteckt leben und mögen daher oft nicht gefunden werden.

\section{Achetidae.}

7. Curtilla gryllotalpa L. Klausen, 1 Expl.

8. Nemobius silvestris F. Überall im Eisacktal unter Steinen, springen äußerst gewandt.

9. Acheta campestris L. Im Eisacktal häufig, dagegen fand ich sie niemals am Gardasee, wo sie - wenigstens am Südwestufer bei Gardone - durch die folgende vertreten zu werden scheint.

10. Gryllus desertus Pall. In Gardone in lichten Olivenhainen zahlreich unter Steinen.

11. Gryllomorpha dalmatina Ocsk. In Gardone zahlreich in Häusern. besonders in Vorratskammern und Kellern.

\section{Tettigoniidae (Locustidae).}

12. Pholidoptera (Thamnotrizon) aptera Fabr. Im Eisacktal, besonders bei Klausen (Thinneschlucht), an bewachsenen Geröllhalden häufig.

13. Chelidoptera (Platycleis) albopunctata Goeze (grisea Fabr.). Klausen, Atzwang. Sehr variabel in Zeichnung und Färbung, oft ganz einfarbig.

14. Decticus verrucivorus L. Klausen, auf üppigen Wiesen, an Geröllhalden.

15. Barbitistes serricauda Fabr. Einige $\sigma \sigma$ von Mitte Juli ab bei Klausen und Atzwang auf Brombeersträuchern.

16. Acrydium (Tettix) kraussi Saulcy. Gardone; bei Cobelli ,Ortotteri del Trentino“ 1886 nebst „Appendice“ 1906 nicht aufgeführt; von Karny zu bipunctatum L. gezogen, neuerdings von $\mathrm{Haij}$ und Krauß als Art aufrechterhalten.

\section{Locustidae (Acridiidae).}

17. Stenobothrus lineatus Panz. Häufig bei Klausen; noch Ende Juli zahlreiche junge Larven vorhanden.

18. Arcyptera (Stethophyma) fusca Pall. Im ganzen Eisacktal sehr häufig. 
19. Omocestus (Stenobothrus) ventralis Zett. (rufipes Zett.) Gardone.

20. Stauroderus (Stenobothrus) bicolor Charp. Klausen, Atzwang.

21. Aeolopus (Epacromia) strepens Latr. Selten; nur wenige abgeflogene Stücke Ende Mai bei Gardone.

22. Oedipoda coerulescens L. Besonders häufig unterhalb Atzwang an der Kaiserstrasse.

23. - miniata Pall. Wie die vorige.

24. Locusta (Acridium) tartarica Fisch. (aegyptium L.) Gardone, Maderno, Sirmione 1. 6.; an Olivenstämmen, auf Rubus, im Gras etc. Fliegt bei Annäherung auf und setzt sich in Baumkronen. Ich fing von Mitte Mai bis Mitte Juni ca. 10-12 Tiere, die fast sämtlich stark abgeflogen waren. Da die Imagines in der dortigen Gegend am häufigsten im September und Oktober sind (man findet um diese Zeit - in der Minderzahl - auch Larven), so möchte ich annehmen, daß im allgemeinen die Überwinterung im ausgebildeten Zustand erfolgt, wenn anders man nicht auf das Auftreten von zwei Generationen schließen will, was jedoch bei der langsamen Entwicklung des großen Tieres unwahrscheinlich ist.

Die 우우 würden also etwa in den Monaten März-Mai Eier legen und die Larven zum größten Teil schon im September erwachsen sein. Die Imagines treten im Oktober auf, überwintern und halten sich in einzelnen Exemplaren bis zum Juni, wo sie dann schon meist abgeflogen sind. Nur diejenigen Larven, die - aus sehr spät abgelegten Eiern stammend nicht bis zum Oktober ihre volle Ausbildung erreichten, überwintern und liefern schon im März die Imagines.

Die im „Prodromus“ von Brunner v. Wattenwyl vorliegende und von T ümpel übernommene Angabe „bereits im März erwachsen, fliegt bis Juni“, wäre also in dieser Weise zu rektifizieren.

25. Podisma alpina Koll. Bei Waidbruck, am Eingang des Grödner Tales (475 m), am Bachrand.

26. Calliptamus italicus Serv. Bei Atzwang (zusammen mit Oedipoda) sehr häufig; darunter die f. marginella Serv.

\section{Lepidoptera.}

\section{Rhopalocera.}

1. Papilio podalirius L. 1 Exemplar in Riva am 20. 5., Frühịahrsgeneration. In Gardone, wo ich am folgenden Tage ankam, war die 1. Generation bereits vorbei; sie war nach Angabe von Dr. Baum daselbst noch wenige Tage vorher sehr häufig gewesen. Am 1. 6. traf ich auf Sirmione bereits die ersten zwei Exemplare der 2. Generation, die hier der f. zanclaeus 
angehört; beide entgingen mir, doch habe ich bereits im Juli 1905 zanclaeus dort sehr zahlreich in den Olivenhainen beobachtet, die er in unstetem Fluge durchzieht. In Gardone trat die 2. Generation bis zum Tage meiner Abreise am 13. 5. nicht auf; ich traf sie erst wieder in Klausen Ende Juli. Die dort fliegende Sommergeneration bildet den Uebergang zu zanclaeus, indem der Leib in bedeutend geringerer Ausdehnung weiß bestäubt ist. Diese Südtiroler Sommerform ist ziemlich klein im Verhältnis zu der vom Südufer des Gardasees. Die Raupe lebt meist an Prunus mahaleb; einmal konnte ich die Eiablage beobachten, die einzeln, gewöhnlich an der Unterseite der Blätter, erfolgte. - Der Falter wird, zuweilen in großen Schwärmen, namentlich an solchen Stellen an der Straße angetroffen, wo sich mit Unrat vermengtes Wasser angesammelt hat.

2. - machaon L. 1 Exemplar der 1. Generation in Riva 20. 5.; 2. Generation im Eisacktal Ende Juli unter den podalirius.

3. Parnassius apollo L. (rubidus Fruhst.). An steinigen Hängen im ganzen Eisacktal häufig; es werden bis in den August und zuweilen noch später frisch geschlüpfte Exemplare angetroffen, was zu der Annahme verleiten könnte, apollo habe zwei Generationen. Dem ist aber bekanntermaßen nicht so; vielmehr mögen die Falter wohl deswegen ungleichmäßig schlüpfen, weil die Puppe oft an Stellen liegt, die der Erwärmung wenig zugänglich sind. Unter der typischen Form kamen Stücke der f. decora Schultz vor, darunter ein besonders auffällig ausgeprägtes $O$ mit reich rot gekernten Analflecken und sehr großen hinteren Ozellen, die unscharf halbiert sind (Kombination mit f. graphica Stich.). Taf. II, Fig. 2.

4. Aporia crataegi L. Gardone, Klausen.

5. Leptidia sinapis L. Klausen, Juli; darunter mehrfach die f. dini= ensis $B$.

6. Colias hyale L. In Gardone und Klausen; etwas größer und namentlich auf der Unterseite lebhafter gefärbt als hiesige Stücke.

7. - edusa F. In Gardone am 21. 5. noch ein abgeschabtes Stück der ersten und am 12. 6. bereits zahlreiche frische Tiere der zweiten Generation. In Klausen die zweite Generation erst im Juli.

8. Limenitis camilla Schiff. In Gardone häufig, Ende Mai meist abgeflogen; in Klausen noch Ende Juli ein frisches $q$. Findet sich nie mit den beiden folgenden zusammen, obwohl ihr dieselben Lokalitäten zusagen.

9. - sibilla L. Im ersten Teil des Grödner Tales sehr häufig.

10. Neptis lucilla F. Zugleich mit der vorhergehenden Art, doch seltener; auch bei Klausen. Beide lieben schattige Partien in der Nähe von Wasserläufen. 
11. Vanessa io L. In Klausen, 1 Exemplar. Ende Juli.

12. - polychloros L. Überall häufig.

13. Polygonia $\mathbf{c}=\mathbf{a l b u m ~ L . ~ K l a u s e n . ~}$

14. Pyrameis atalanta L. Gardone, Klausen.

15. - cardui L. Gardone.

16. Melitaea phoebe Knoch. Klausen, häufig; ein Stück albinotisch.

17. - didyma meridionalis Stdgr. In Gardone die häufigste Melitaea; am 20. Mai schon viele abgeflogene Stücke. In Klausen (Thinneschlucht, Sternklamm b. Villnöß) ebenfalls häufig, doch erst Ende Juli. Die $\sigma \sigma$ recht variabel, feurig rot gefärbt; 우오 sehr viel seltener und ebenfalls sehr variabel in Farbe und Größe. In Gardone ein $q$ mit rotgelber Grundfarbe auf allen Flügeln; in Klausen das größte Stück mit 4,8 cm Flügelspannung, das kleinste mit $3,6 \mathrm{~cm}$; letzteres auf den grünlichgrauen Vfl. mit zahlreichen eingesprengten rotgelben Flecken.

18. - athalia Rott. Klausen.

19. - aurelia Nick. Gardone, 1 Exemplar.

20. Argynnis daphne Schiff. Klausen, Villnöß; namentlich in den Seitenschluchten des Eisacktales häufig; Juli.

21. - Iatonia L. In Gardone ein Exemplar von feurigroter Farbe (wie M. didyma $\sigma^{\text {) }}$, in Klausen häufiger, in der typischen Färbung.

22. - niobe L., f. eris Meig. Klausen, 1 Exemplar.

23. - adippe L. Klausen, häufig. Unter den 우오 1 Expl. von $6,3 \mathrm{~cm}$ Flügelspannung.

24. - aglaja L. Klausen, 1 Exemplar.

25. - paphia L. Klausen, ziemlich häufig; die f. + valesina Esp., die zuweilen in der Thinneschlucht häufig ist, nicht beobachtet.

26. Melanargia galatea procida Herbst. Gardasee, Eisacktal; sehr häufig.

27. Erebia nerine Fr. An den Felswänden der Mendel bei Bozen sehr häufig.

28. Satyrus hermione L. Von Ende Juni an im Eisacktal an der Straße ziemlich häufig; setzt sich gern an die Stämme der echten Kastanien. Auf Sirmione am 1. 8. 05 in den Olivenhainen zahlreiche 우우.

29. - semele L. Gemein im Eisacktal in großen, dunkler und feuriger als bei uns gefärbten Stücken. Erscheint bereits 2 bis 3 Wochen vor hermione.

30. - statilinus allionia F. 1. 8. 04, auf Sirmione in den Olivenhainen zusammen mit hermione häufig.

31. - actaea cordula F. Von Ende Juli an besonders an den steinigen Abhängen des Eisacktals; die Hauptform bei Torbole am Gardasee. 
32. Satyrus dryas Sc. Anfang August traten die ersten $\sigma \sigma$ auf; die Hauptflugzeit erst Ende August. An denselben Örtlichkeiten wie cordula. - Die Satyrusarten treten also im Eisacktal in der Reihenfolge semele, hermione, cordula, dryas auf; die 우우 erscheinen durchweg $1-2$ Wochen später als die $\sigma \sigma$.

33. Pararge egeria egeria L. Gardone, vereinzelt.

34. - megaera L. Gardone, Sirmione, Klausen; ziemlich häufig.

35. - hiera F. Gardone, Klausen; ziemlich selten, namentlich im weiblichen Geschlecht.

36. Epinephele jurtina L. Im Gardaseegebiet und im Eisacktal gemein; in beiden Geschlechtern sehr variabel (Übgg. zur f. hispulla $\mathrm{Hb}$.).

37. - tithonus L. Bei Desenzano am 1. 8. 04 sehr häufig.

38. Coenonympha arcania L. Gardone, Klausen; häufig.

39. - pamphilus L. Gardone, Klausen; häufig.

40. Libythea celtis Laicharting-Fueß1. Gardone, Anfang Juni; in Klausen erst Ende des Monats; sehr gemein.

41. Thecla w.=album Kn. Atzwang, 1 Exemplar.

42. - ilicis Esp. Gardone, Klausen; häufig. Die f. cerri Hb. nicht selten unter der Hauptform.

43. Zephyrus quercus L. In Gardone in den niedrigen Eichenbeständen oberhalb der Olivenregion; in Klausen 1 .

44. Chrysophanus alciphron gordius Sulz. Bei Klausen (Sternklamm, Thinneschlucht) ziemlich häufig; 1 Expl. bildet den Übergang zur f. confluens Courv. Taf. II, Fig. 3.

45. Ch. phlaeas L. Klausen.

46. Lycaena argus L. Klausen; 1 Expl.

47. - argyrognomon Bergstr. Klausen.

48. - baton Bgstr. Gardone.

49. - orion Pall. In Riva am 20. 5. auf der Ponalestraße sehr häufig; ferner in Gardone vereinzelt. In Klausen erst Ende Juni. Die 오오 meist der f. nigra Rühl angehörig.

50. - icarus Rott. Gardone.

51. - amandus Schn. Bei Klausen, Waidbruck in sehr großen Exemplaren $\left(\sigma \sigma^{\prime}\right)$ mit breitem dunklem Rand. (Flügelsp.: $3,9 \mathrm{~cm}$.) Taf. II, Fig. 4.

52. - hylas Esp. Klausen, sehr vereinzelt; Ende Juli.

53. - meleager Esp. Klausen, in der zweiten Julihälfte; die $\sigma \sigma$ spärlich; darunter 1 Exemplar der f. steevenii Tr.

54. - bellargus Rott. In Gardone die häufigste L., Mai; die $\sigma^{\top} \sigma^{\top}$ variieren ungemein, neben solchen, die auf allen Flügeln blau bestäubt sind, finden sich Exemplare, die nur um die Augenflecken wenige blaue Schüppchen zeigen.

55. - jolas O. Herr Postverwalter Frank-Passau dedizierte mir ein Expl. aus Blumau (südl. Atzwang), wo die Art häufiger ist. 
56. Lycaena cyllarus $\mathrm{f}$. $q$ andereggii Rühl. Gardone, häufig; scheint dort Lokalform zu sein. $\sigma \sigma$ nicht beobachtet.

57. - minima FueßıI. Gardone; $\sigma$ ziemlich stark blau bestäubt.

58. Cyaniris argiolus L. Klausen, ziemlich häufig.

\section{Netrocera.}

1. Adopaea thaumas Hufn. Klausen.

2. Carcharodus lavaterae Esp. Im ganzen Gardaseegebiet in teils helleren, teils dunkleren Stücken; häufig bei Malcésine, Cassone 16. 6.

3. Scelothrix carthami Hb. In sehr intensiv gezeichneten Stücken, bei Malcésine 16. 6.

4. Pyrgus sao Hb. Klausen, häufig.

5. Macroglossa stellatarum L. Überall; sehr gemein Ende Juli 1905 auf Sirmione, an Salvia schwärmend.

6. - fuciformis L. (=Hem. scabiosae Z.). Klausen, vereinzelt.

7. Choerocampa elpenor L. Gardone, Klausen.

8. Deilephila vespertilio Esp. Zwei Raupen bei Atzwang an Epilobium rosmarinifolium; die eine, fast erwachsen, entgegen der sonstigen Gewohnheit der Raupe bei Tage an der Futterpflanze (nach D a n n e h 1 sind solche Raupen meist angestochen), ließ sich beim Näherkommen fallen. Sie lieferte eine anscheinend gesunde, jetzt (18. 3. 11) noch lebende Puppe. - Im Freien lebt die Raupe bekanntlich nur an Epilobium rosmarinifolium; sie nehmen auch in der Gefangenschaft - außer bei der Zucht aus dem Ei - niemals eine andere Epilobium-Art an. Der Falter schwärmt gern an Saponaria.

9. - euphorbiae L. Klausen.

10. Daphnis nerii L. 1 Exemplar an einer Mauer bei Salò; zuweilen ziemlich häufig, auch bei Klausen.

11. Protoparce convolvuli L. Klausen.

12. Acherontia atropos L. In ganz Südtirol meist gemein; dieses Jahr die Raupen sehr spärlich.

13. Dilina tiliae L. Ein großes dunkelgrünes Exemplar, mit schwärzlich bestäubten Hfl., bei Waidbruck (Eingang des Grödner Tals); frischgeschlüpft an einer feuchten Felswand.

14. Dicranura erminea Esp. In Venedig auf dem Lido in einer Pappelallee, am 31. 7. 05, gemein.

15. Exaereta ulmi Schiff. Bei Atzwang die Raupe zuweilen in großen Mengen an Ulmus.

16. Thaumetopoea pityocampa Schiff. In Gardone und Klausen zahlreiche Nester an Pinus.

17. Saturnia pyri Schiff. Gardone; zahlreich von Ende Juni ab, gegen 11 Uhr um das elektrische Licht flatternd. 1905 wurde 
mir noch am 1. August in Sirmione von einem Dorfbewohner ein lebendes Stück gebracht.

18. Saturnia pavonia L. Einige Raupen in Klausen an Salix.

19. Aglia tau L. $1 \sigma^{\prime}$ am 20. 5. in Riva.

20. Eriogaster lanestris L. Klausen; die Nester zahlreich an Birken; Ende Juli waren viele Raupen noch nicht erwachsen.

21. Lasiocampa quercus $\mathrm{L}$.

22. - trifolii Esp. Von beiden Arten am Gardasee zahlreiche Raupen, wahrscheinlich aber von Lokalformen derselben; erstere zuweilen an Lorbeer.

23. Macrothylacia rubi L. Ein + , größer und dunkler als bei uns, in Gardone di sopra.

24. Odonestis pruni L. Venedig (Lido); Gardone, am Licht.

25. Acronicta megacephala F. Klausen, 1 Exemplar.

26. Agrotis janthina Esp. Gardone, an Lindenblüten, 1 Exemplar.

27. - pronuba L. Gardone, 1 Exemplar.

28. - comes Hb. Gardone, häufig.

29. - simulans Hufn. Gardone, groß.

30. - saucia $\mathrm{Hb}$. Gardone.

31. Mamestra genistae Bkh. Gardone, am Köder.

32. Dianthoecia luteago $\mathrm{Hb}$. Gardone, Klausen; am Licht.

33. Bryophila perla F. Klausen, häufig.

34. Valeria oleagina F. Raupe an Schlehen, bei Atzwang.

35. Rhizogramma detersa Esp. Klausen, häufig an Zäunen etc.

36. Leucania $\mathbf{l}=\mathbf{a l b u m} \mathrm{L}$. Gardone.

37. - evidens $\mathrm{Hb}$. Gardone, 1 Exemplar.

38. Calophasia lunula Hfn. Gardone, sehr häufig.

39. Acontia luctuosa Esp. Gardone.

40. Prothymia viridaria $\mathrm{Cl}$. Am Abhang des Mte. Castello bei Gaïno.

41. Emmelia trabealis Sc. Gardone.

42. Plusia chalcytes Esp. Gardone, 1 Exemplar.

43. - gamma L. Klausen, sehr dunkel gefärbt.

44. Euclidia glyphica L. Gardone.

45. Grammodes algira L. Je 1 Exemplar in Fasano bei Gardone und in Klausen.

46. Catocala electa Bkh. Ende Juni 2 erwachsene Raupen an Zweigen von Salix; Klausen. Ergaben Anf. August die Falter.

47. - puerpera Giorna. Klausen, Waidbruck, Blumau; Raupen an dürren, niedrigen Pappelbüschen und einer schmalblättrigen Weide. Freilandraupen nehmen in der Gefangenschaft keine andere Nahrung an. Ergaben Mitte August die Falter.

48. - conversa agamos $\mathrm{Hb}$. In Klausen, 1 Expl., frischgeschlüpft an einer Felswand. 
49. Apopestes spectrum Esp. Gardone; 30. 5. eine Raupe $\left(1^{1 / 2} \mathrm{~cm}\right)$ an Genista (Abhänge des Mte. Bartholomeo).

50. Herminia crinalis $\mathrm{Tr}$. Klausen, 1 우.

51. Hypena obsitalis $\mathrm{Hb}$. Gardone; namentlich in altem Gemäuer, Höhlen etc. sehr häufig.

52. - antiqualis Hb. 1 Expl. am Mte. Bartholomeo bei Gardone.

53. Geometra papilionaria L. Waidbruck.

54. Acidalia filicata $\mathrm{Hb}$. Gardone, häufig.

55. - degeneraria $\mathrm{Hb}$. Gardone.

56. - luridata Z., f. confinaria H.-S. Gardone, häufig. Neu für Oberitalien.

57. Codonia annulata Schulze. Gardone.

58. Rhodostrophia calabraria Zell. Gardone, Ende Mai; häufig.

59. Ortholitha bipunctaria W. V, Klausen.

60. Larentia tophaceata W. V. Gardone, in höheren Lagen.

61. - hydrata Tr. Gardone.

62. Phibalapteryx tersata Schiff. Gardone.

63. Venilia macularia L. Gardone, in sehr intensiv gefärbten Stücken, häufig.

64. Boarmia gemmaria Brahm. Gardone.

65. Gnophos furvata Schiff. $1 \div(5,3 \mathrm{~cm})$ in Klausen.

66. Phasiane glarearia Brahm. Gardone, häufig.

67. Scodiona conspersaria F. Gardone.

68. Syntomis phegea L. In Gardone mit f. pfluemeri Wacquant, in Klausen mit f. iphimedia Esp.; ein Exemplar auf der linken Seite ganz schwarz, mit nur einem weißen Punkt auf dem Hinterflügel, auf der rechten Seite normal gezeichnet. Taf. II, Fig. 6. (Vgl. Intern. Ent. Zeitschr., Guben, 4. Jahrg. p. 182, wo die betr. Tiere auch abgebildet sind.)

69. Dysauxes punctata F. Gardone; in der zweiten Hälfte des Mai sehr häufig; namentlich um Rosa canina schwärmend und an der Unterseite der Blätter sitzend.

70. Spilosoma mendica $\mathrm{Cl}$. Gardone, 1 Expl. (ㅇ).

71. Phragmatobia fuliginosa L. Gardone.

72. Arctinia caesarea Goeze. Gardone, 2 Expl.

73. Arctia villica L. In Gardone sehr häufig mit f. angelica B. ( 2 typ. $\sigma \sigma$ ); in Klausen seltener. Fliegt oft bei Tage und kommt abends ans Licht.

74. Callimorpha dominula f. insubrica Wackerzapp. Klausen, Waidbruck; nicht selten. Taf. II, Fig. 5.

75. - quadripunctaria Poda. Von Ende Juli an im Eisacktal häufig, der Falter namentlich auf Eupatorium cannabinum; die hier fliegende Form $(30-32 \mathrm{~cm})$ bildet den Übergang zur subsp. magna Spul. 
76. Coscinia cribrum L. Im unteren Eisacktal in den ff. punctigera Frr. und candida Cyr.; die Hauptform kommt nicht vor. Der Falter sitzt gern in Berberitzensträuchern; die Raupe ist leicht mit Salat etc. zu erziehen.

77. Hypocrita jacobaeae L. Häufig am Gardasee und namentlich im Eisacktal.

78. Endrosa kuhlweini $\mathrm{Hb}$. Atzwang, $1 \sigma$.

79. Lithosia pallifrons $Z$. Gardone, ein typisches $q$.

80. Anthrocera purpuralis Brünnich. Klausen.

81. - achilleae Esp. Gardone. Mitte Mai die häufigste Art.

82. - Ionicerae Esp. Klausen; sehr große Form.

83. - filipendulae L. Gardone, Klausen; an letzterem Orte sehr groß.

84. - angelicae O. Gardone, 1 Expl.; f. cingulata Dziurz. Klausen, 1 Expl.

85. - ephialtes L., f. trigonellae Esp. Klausen, Waidbruck (Grödner Tal); von Ende Juli ziemlich häufig.

86. - transalpina Esp. Gardone, Klausen; an letzterem Orte häufiger.

87. Procris manni heydenreichii Ld. Gardone, in der zweiten Maihälfte häufig.

88. Cochlidion limacodes Hufn., f. bufo L. Gardone, 1 Expl.

89. Psyche unicolor Hufn. Klausen.

90. Apterona crenulella, f. $q$ helix Sieb. In Gardone und Klausen, an Häusern und Chausseesteinen sehr häufig.

91. Crambus craterellus Sc. In Gardone, in sehr intensiv gefärbten Stücken, häufig.

92. Hypochalcia hepaticella Rag. Ende Mai in Gardone sehr häufig; bisher nur aus Meran bekannt.

93. Salebria semirubella Sc. Gardone, häufig.

94. Hypsopygia costalis F. Gardone.

95. Stenia punctalis Schiff. Gardone.

96. Scoparia crataegella $\mathrm{Hb}$. Gardone, 2 Expl.

97. Pionea forficalis L. Riva.

98. Euergestis extimalis Sc. Gardone.

99. Pyrausta cespitalis Schiff. Gardone.

100. - aurata Sc. Klausen.

101. Dyspessa ulula Bkh. Gardone, 1 Expl. geschöpft.

102. Zeuzera pyrina L. Torbole 16. 6., am Licht.

103. Sesia stomoxyformis $\mathrm{Hb}$. Gardone; 1 Exemplar an blühendem Liguster.

104. - empiformis Esp. Nago 17. 6., Klausen; je 1 Expl.

105. - chrysidiformis Esp. Gardone, Nago; namentlich am ersteren Orte Anfang bis Mitte Juni ziemlich häufig; der Falter sitzt zu jeder Tageszeit meist an den Spitzen der Ampferpflanzen und Grashalme und ist sehr flüchtig. 
106. Adela degeerella L. Klausen (Thinneschlucht).

107. Thyris fenestrella. Salò, 9. 6.; Nago-Torbole, 16. 6.; Klausen.

An Pfützen, auf Eupatorium, Umbelliferen etc.

\section{Diptera.}

\section{Bibionidae.}

1. Bibio hortulanus L. Riva, 20. 5.

2. - marci L. Kufstein, 20. 5.

\section{Tipulidae.}

3. Ctenophora festiva Meig. Gardone, 1 Expl.

4. - pectinicornis L. Klausen.

5. Tipula maxima Pora. Gardone, Klausen.

6. Pachyrhina crocata L. Gardone.

\section{Stratiomyidae.}

7. Ephippiomyia ephippium Fabr. Klausen, 1 Expl., träge an einer Weide sitzend.

8. Odontomyia (Hoplodonta) viridula Fabr. Gardone.

9. Chloromyia formosa Scop. Gardone.

10. - melampogon Zell. Sirmione, 31. 5., Gardone, Klausen.

\section{Tabanidae.}

11. Tabanus ater Rossi. Gardone, Klausen; häufig.

12. - autumnalis L. Sirmione, 1. 6.

13. - nemoralis Meig. Gardone.

\section{Leptidae.}

14. Leptis tringaria L. Gardone.

15. Chrysopilus auratus Fabr. Gardone, an Bachrändern.

\section{Asilidae.}

16. Dioctria bicincta Meig. Gardone.

17. - hyalipennis Fabr. Sirmione, 31. 5.

18. Dasypogon teutonus L. Klausen; ungemein häufig, sehr räuberisch.

19. Habropogon appendiculatus Schin. Gardone.

20. Laphria flava L. Klausen.

21. - fulva Meig. Klausen, 1 Expl.

22. Andrenosoma albibarbe Meig. Klausen, 1 Expl.

23. - atrum L. Klausen, 1 Expl.

24. Pamponerus (Asilus) germanicus L. Klausen.

25. Machimus colubrinus Meig. Gardone.

26. - rusticus Meig. Klausen. 


\section{Bombyliidae.}

27. Leucamoeba aethiops Fabr. Gardone, Malcésine, 16. 6.; häufig.

28. Argyramoeba trifasciata Meig. Gardone.

29. - anthrax Schik. Klausen.

30. Molybdamoeba tripunctata Wied. Klausen.

31. Anthrax fenestratus Fall. Gardone, 1 Expl.

32. - hottentottus L. Gardone, Klausen; häufig.

33. - velutinus Meig. Auf der Chaussee Salò-Gardone sehr häufig. 9. 6.

34. Lomatia sabaeus Fabr. Gardone, sehr gemein.

35. Bombylius ater Scop. Gardone, Malcésine, 16. 6.

36. - fimbriatus Meig. Riva, 20. 5., 1 Expl.

37. - fulvescens Meig. Gardone.

38. - medius L. Gardone, 1 Expl.

39. - venosus Mikn. Gardone; sehr häufig, an Silene schwärmend.

\section{Therevidae.}

40. Scionophora kollari Egg. Gardone.

\section{Scenopinidae.}

41. Scenopinus fenestralis L. Klausen.

\section{Empidae.}

42. Brachystoma vesiculosum Fabr. Gardone.

43. Syneches muscarius Fabr. Sirmione, 31. 5.; häufig.

\section{Syrphidae.}

44. Chilosia illustrata Harr. Klausen; Ende Juli auf Umbelliferen sehr häufig.

45. Xanthogramma ornatum Meig. Gardone, Klausen.

46. Volucella bombylans L. Klausen.

47. - inanis L. Klausen, 1 Expl.

48. - pellucens L. Klausen.

49. - zonaria Poda. Gardone; häufig, namentlich auf blühendem Ligustrum vulgare.

50. Eristalis tenax L. Gardone.

51. - arbustorum L. Klausen.

52. Helophilus trivittatus Fabr. Torbole, 16. 5.; gemein am Seeufer.

53. Myiatropa florea L. Gardone, Klausen.

54. Merodon albifrons Meig. Klausen, 1 Expl.

55. Myiolepta luteola Gmel. Salò, 9. 6.

56. Spilomyia diophtalma L. Klausen.

57. Chrysotoxum bicinctum L. Gardone, Klausen.

58. - cautum Harr. Gardone, Klausen.

59. - festivum L. Gardone, 1 Expl. 


\section{Tachinidae.}

60. Echinomyia ferox Panz. Klausen.

61. - magnicornis Zett. Salò, Klausen. 1 Exempl. mit vollständig rotgelbem Abdomen, ohne schwarze Binde, bei Klausen.

62. Sphyrocera sphyrocera (Macq.) Bezzi. Klausen.

63. Rhynchista prolixa Meig. Gardone.

64. Ocyptera brassicaria Fabr. Gardone.

65. Cylindromyiopsis sanguinea Rond. Gardone, 1 Expl.

66. Mintho praeceps Scop. Gardone.

67. Dexia rustica Fabr. Klausen.

68. Lucilia sericata Meig. Gardone.

69. Melanophora roralis L. Salò, 9. 6., Klausen.

70. Phasia crassipennis Fabr. Salò, Gardone; auf Umbelliferen.

71. Helomyia lateralis Meig. Gardone.

\section{Scatomyzidae.}

72. Scatophaga stercoraria L. Gardone.

\section{Anthomyidae.}

73. Graphomyia maculata Scop. Gardone.

74. Stomoxys calcitrans L. Gardone.

\section{Sciomyzidae.}

75. Limnia unguicornis Scop. Gardone.

76. Coremacera marginata Fabr. Gardone.

\section{Ortalidae.}

77. Platystoma seminationis L. Malcésine, 16. 5.; häufig, auf faulendem Holz.

\section{Trypetidae.}

78. Oxyphora flava Geoffr. Salò, 9. 6.

Conopidae.

79. Physocephala vittata Fabr. Klausen.

80. Sicus ferrugineus L. Klausen.

\section{Hymenoptera.}

Apidae.

1. Apis mellifica f. ligustica Spin. Vertritt im ganzen Gardaseegebiet die Stammform; letztere im Eisacktal (Klausen).

2. Bombus lapidarius L. Bei Malcésine, 15. 6., nur diese Art sehr häufig beobachtet; 1 Exemplar in Gardone.

3. - pratorum L. Klausen.

4. - terrestris L. Gardone, ziemlich kleine Form.

5. - hortorum L. 2 우 우 aus Gardone; zahlreiche of der f. argillacea Scop. im Gardaseegebiet und bei Klausen.

6. Bombus agrorum, f. pascuorum Sc. Klausen, Salò; an letzterem Orte sich mehr der Stammform nähernd. 
7. Bombus variabilis Schmdk. Klausen; hier häufiger durch die f. notomelas Krchb. vertreten; in Gardone die zur f. tristis hinführende f. propenigra Vogt.

8. - elegans Seidl. Klausen, häufig.

9. - soroënsis, f. sepulcralis Schmdk. Bei Gardone der häufigste Bombus; 1 Expl. zur f. proteus Gerst. gehörig.

10. Antophora parietina F. f. schenki D. T. Klausen, 1 Expl.

11. - crinipes Sm. Gardone; ziemlich häufig an Silene schwärmend.

12. - acervorum L. Riva, 20. 5., häufig; Klausen; in Gardone 1 Exemplar der f. nigra Fr.

13. - balneorum Lep. f. obesa Gir. Klausen, 1 Expl.

14. Macrocera ruficollis Brullé. Riva, 20. 5., 1 Expl.

15. Eucera nitidiventris Mocs. Bei Sirmione auf einer feuchten Wiese am Seeufer zwei Schwärme von je 40-50 б $\sigma$, eng zusammengeballt an zwei benachbarten Halmen (Spätnachmittag).

16. Xylocopa violacea L. Überall am Gardasee häufig, namentlich an Ginster schwärmend; in Klausen nicht beobachtet.

17. Ceratina cucurbitina Rossi. Riva, 20. 5., 1 Expl.

18. Rophites 5=spinosus Spin. Gardone, 1 Expl.

19. Andrena taraxaci Giraud. Riva, 20. 5., 1 Expl.

20. Megachile melanopyga Costa. Gardone, 1 Expl.

21. - argentata F. Gardone, 1 Expl.

22. Chalicodoma muraria F. Überall häufig; die Bauten an den Felswänden.

23. Osmia bicolor Schrk. Riva, 20. 5., 1 Expl.

24. - rufohirta Latr. Am Gardasee häufig.

25. Anthidium 7=dentatum Latr. Riva, sehr häufig; (Ponalestraße). 1 Expl. in Gardone.

26. - punctatum Latr. Gardone, Klausen.

27. Psithyrus rupestris F. 1 in Malcésine.

28. - barbutellus K. Mit der f. maxillosa Klug in Klausen häufig.

29. Melecta armata, f. aterrima Lep. 1 Expl. dieser seltenen Form in Riva, 20. 6.

30. Nomada succincta $\mathrm{Pz}$. Riva, 20. 5.

\section{Crabronidae.}

31. Ammophila heydeni Dahlb. Klausen.

32. Psammophila hirsuta Sc. Torbole, 17. 6.

33. Sceliphron destillatorius Ill. Klausen, 1 Expl.

34. Crabro cribrarius L. Klausen.

\section{Vespidae.}

35. Vespa crabro L. Überall.

36. - germanica F. Gardone. 
37. Polistes gallicus L. Sehr variabel; in Sirmione am Seeufer (an Zäunen), mit viel Gelb und scharf gezeichnet; ebenso in Klausen, doch größer. Sehr kleine und matt gefärbte Exemplare aus Riva.

38. Lionotus punctifrons Thom. Gardone. Bisher wohl nur aus der Südschweiz bekannt geworden.

39. Eumenes coarctatus L. Klausen.

\section{Chrysididae.}

40. Stilbum cyanurum, f. calens F. 1 m unteren Eisacktal, zuweilen nicht seIten. Gern auf Eupatorium cannabinum.

41. Chrysis ignita L. Gardone.

42. - dichroa Dahlb. Gardone, 21. 5., 1 Expl.

\section{Scoliidae.}

43. Discolia hirta Schrank. Klausen; vereinzelt die f. unifasciata F.

44. Triscolia flavifrons F. Diese stattliche Scoliide, deren Larve bekanntlich bei Oryctes nasicornis schmarotzt, traf ich von Ende Mai in Gardone zunächst vereinzelt auf Thymus, dann in großer Menge an Deutzia. Ein Exemplar mit roten Haaren auf dem letzten Hinterleibssegment bildet einen Übergang zu der mehr im südlichen Italien und in Griechenland heimischen f. haemorrhoidalis Fabr.

\section{Mutillidae.}

45. Mutilla maura F. 2 우, Klausen.

\section{Formicidae.}

46. Camponotus ligniperda Latr. Gardone, Klausen.

47. - pubescens F. Gardone, sehr häufig.

48. - maculatus, f. aethiops Varone bei Riva.

49. Formica rufa, f. pratensis Retzius. Klausen.

50. Cremastogaster scutellaris $\mathrm{Ob}$. Gardone.

\section{Chalcididae.}

51. Leucospis dorsigera F. Klausen, auf Umbelliferen.

\section{Evaniidae.}

52. Gasteruption pedemontanum Tourn. Torbole, 11. 6.

\section{Braconidae.}

53. Iphiaulax impostor Scop. Klausen, 17. 6.

Ichneumonidae (Prof. Dr. R. Krieger-Leipzig).

54. Trogus exaltatorius $\mathrm{Pz}$. Klausen.

55. - lutorius F. Klausen.

56. Ichneumon similatorius F. Salò, Klausen.

57. Coelichneumon lineator F. 1 우, Gardone. 
58. Melanichneumon erythraeus Gr. $1 \sigma$, Gardone.

59. Hoplismenus pica Wesm, $\sigma$. Bisher unbekannt. Das Stück stimmt bis auf die Abweichungen, die sich aus der Verschiedenheit des Geschlechts ergeben, und einige kleine Färbungsabänderungen, wie sie auch sonst häufig vorkommen, vollständig zu Wesmaels Beschreibung in den Bulletins de l'Academie de Bruxelles, XXII., 2. P. 1855, p. 406.

Die Abweichungen sind: Am Kopf sind die Oberlippe, die Seiten des Kopfschildes und des Gesichts (diese unten breiter), sowie ein längliches Fleckchen am Augenrande neben den Fühlerwurzeln weiß. Fühlerschaft unten mit weißem Fleck. Der weiße Fühlerring nimmt das 9.-14. Geißelglied ein, das 9. Glied ist unten, das das 13. und 14. oben dunkel, das 8. Glied hat unten, das 15. oben ein helles Fleckchen. Auf der Schwiele unter den Vorderflügelwurzeln nur ein rundliches Fleckchen und am Schildchen nur die hintere Abdachung weiß. Die Dornen des Mittelsegments nur oben am Ende mit weißem Fleck. Vorderhüften unten und Mittelhüften unten an der Spitze weiß. Auch die Schienen und Tarsen der Mittelbeine sind vorn hell gelbbraun. Erstes Hinterleibssegment ganz schwarz, das zweite ohne helle Querlinie vor dem Hinterrande, nur mit weißen Flecken in den Hinterecken.

Die hellen Zeichnungen sind also, wie gewöhnlich beim $\sigma^{\prime}$ am Kopfe und an den vorderen Beinen stärker, im übrigen aber etwas schwächer entwickelt als bei dem Wesmaelschen 오.

Die mittleren Fühlergeißelglieder sind nach innen zu in der Mitte rundlich erweitert, das 9.-18. außen mit Längsschwielen versehen.

Gefangen in Klausen, Juli. Das Stück befindet sich jetzt in der Sammlung des Herrn Prof. Dr. R. Krieger.

60. Mesostenus ligator Gr. 1 오, Klausen.

61. Stenaraeus grammicus Gr. 1 , Gardone.

62. Hemiteles rubrotinctus Thms. (?). 1 우, Klausen.

63. Pimpla instigator F. 1 , Klausen.

64. Ophion luteus L. 1 오, Klausen.

65. Opheltes glaucopterus L. Klausen.

66. Cymatoneura undulata Gr. 2 우, Gardone.

67. Nototrachys foliator F. $1 \sigma$, Gardone.

68. Charops decipiens Gr. $1 \sigma$, Gardone.

69. Agrypon anomelas Gr. $1 \sigma$, Malcésine, 16. 6. 
70. Banchus falcatorius F. Klausen.

71. Rhyssa persuasoria L. Seis, $1002 \mathrm{~m}$ (Fabrikdirektor J. SchwickerLeipzig, leg.).

\section{Siricidae.}

72. Xeris spectrum L. Klausen (Thinneschlucht).

Tenthredinidae (Dr. E. Enslin-Fürth).

73. Cimbex lutea L. (?). Torbole, 16. 6. Möglicherweise auch zu capreae Kon. oder fagi Zadd. zu stellen; die Artzugehörigkeit ist jedoch nicht sicher festzulegen, da die Säge abgebrochen ist.

74. Abia sericea L. Salò.

75. Megalodontes cephalotes F. Klausen.

76. Hylotoma berberidis Schrk. Klausen.

77. - rosae L. Salò.

78. Monophadnus n. sp.? Das Stück (우) hat einen schmalen Wangenanhang, d. h. die Augen berühren die Mandibelbasis nicht. Wegen der übrigen Merkmale könnte man es zu Ardis, Rhadinoceraea oder Monophadnus stellen. Zu Ardis paßt es aber wegen der Bildung der Fühler und der Sägescheide nicht und zu Rhadinoceraea kann es wegen der Fühlerbildung ebenfalls nicht gehören. Am besten stimmt es noch mit Monophadnus (longicornis Htg.) überein, hier soll aber eigentlich ein Wangenanhang nicht vorhanden sein. Vermutlich ist es eine n. sp., doch ist diese nach dem einen Stück nicht sicherzustellen. Klausen, 20. 5 .

79. Macrophya rustica L. Salò, Klausen.

80. - annulata Geoffr. 1 우, Klausen.

81. - 4=maculata f. poecilopus Aich. Riva, 20. 5.

82. - punctum=album L. Gardone.

83. Tenthredopsis dorsalis Lep. Riva, 20. 5.

84. - litterata f. cerasi L. Klausen.

85. - sordida Hl. Gardone.

86. Tenthredo flava Poda. Klausen.

87. - albicornis F. Klausen.

\section{Rhynchota.}

\section{Hemiptera heteroptera.}

\section{Pentatomidae.}

1. Eurygaster maura L. Gardone, Klausen. Meist an Getreideähren, deren Körner sie ansaugt. Darunter auch die f. picta Fab. und Übergänge zur f. nigra Fieb.

2. - hottentotta H.-S. 1 Expl., Klausen. 
3. Graphosoma lineatum L. Klausen, 2 Expl. auf Umbelliferen.

4. Cydnus nigrita Fab. Sirmione, 31. 5., wie die folgende, oft an Hauswänden emporkriechend.

5. Sehirus maculipes M. R. Sirmione, 31. 5.

6. Aelia rostrata Boh. Gardone.

7. - virgata Kl. Gardone, häufiger als vorige, gern an Getreideähren.

8. Stagonomus bipunctatus F. Gardone, 1 Expl.

9. Eusarcoris melanocephalus F. Gardone, 1 Expl.

10. Staria lunata Hahn. Überall im Gardaseegebiet.

11. Peribaius sphacelatus F. Gardone, 1 Expl.

12. Carpocoris fuscispinus Boh. Klausen.

13. - nigricornis F. Gardone, Klausen; sehr häufig.

14. Dolycoris bacc arum L. Wie die vorige, doch seltener.

15. Palomena prasina Fall. Klausen; an Rubus, Alnus etc.

16. Piezodorus incarnatus Germ. f. alliacea Germ. Gardone, 1 Expl.

17. Raphigaster griseus Fab. f. impunctata Garbl. Klausen, 1 ExpI.

18. Tropicoris rufipes $\mathrm{L}$. Überall.

19. Eurydema decoratum H.-S. 1 Expl.

20. - ornatum L.

21. - oleraceum L. Alle drei Arten zusammen an einer Crucifere gefunden, Gardone.

22. Pinthaeus sanguinipes Fab. 1 Exemplar dieser überall äußerst seltenen Art in Klausen am Wege gefunden; aus Tirol wohl bisher noch nicht bekannt (cf. Hüeber).

23. Picromerus bidens L. Klausen, häufig.

24. - nigridens Fab. 1 Exemplar dieser seltenen Art in Klausen.

\section{Coreidae.}

25. Syromastes marginatus L. Überall häufig; in Salò, 9. 6., ein auffallend kleines, rötlich schimmerndes Exemplar.

26. Verlusia rhombea L. Torbole, 17. 6., 1 Expl.

27. Gonocerus juniperi Fab. Gardone, 1 Exemplar an Juniperus phoeniceus.

28. Loxocnemis dentator Fab. Selten unter der folgenden Art.

29. Coreus denticulatus Scop. Gardone, zahlreich geschöpft.

30. Camptopus lateralis Ger. Auf blumigen Hängen des Mte. Bartolomeo bei Gardone häufig geschöpft.

31. Stenocephalus agilis Scop. Klausen; zahlreich (auch Larven) unter Steinen; erst Ende Juli erwachsen.

32. Therapha hyoscyami L. Gardone, Klausen; vereinzelt, ist sehr flüchtig.

33. Corizus capitatus Fab. Riva, 31. 5., Sirmione, 31. 5.; 2 Expl.

34. - crassicornis L. Gardone, 1 Expl.

35. - parumpunctatus Schil. Gardone, 1 Expl. 


\section{Lygaeidae.}

36. Lygaeus familiaris F. Nur an einer Stelle bei Klausen in Anzahl gefunden, meist an Vincetoxicum.

37. - equestris L. Mit der folgenden in Gardone in lichten Olivenhainen, namentlich an steinigen, bewachsenen Stellen; auch in Klausen.

38. - apuanus Rossi. Nur in Gardone, cf. No. 37.

39. Peritrechus luniger Schik. Gardone, 1 Expl.

40. Aphanus pini L. Klausen, 1 Expl.

41. - phoeniceus Rossi. Am Mte. Castello bei Toscolano-Gaïno in 1 Exemplar, 10. 6.

42. Beosus luscus Fab. Gardone, Sirmione, 31. 5.

43. Emblethus verbasci Fab. Gardone, 1 Expl.

44. Pyrrhocoris marginatus Kol. Klausen.

Phymatidae.

45. Phymata crassipes Fab. Gardone, Toscolano, Klausen; auf Umbelliferendolden, wo sie auf andere Insekten Jagd macht.

\section{Hydrometridae.}

46. Velia currens F. Gardone; zahlreich auf einem Bergbach in der Barbaranoschlucht.

\section{Reduvidae.}

47. Oncocephalus squalidus Rossi. Kam in Gardone im Hause ans Licht geflogen (2 Expl.); die Larve fand ich unter einem Stein an derselben Örtlichkeit wie Lygaeus equestris (s. das.), in 1 Expl. Sie produziert ein klebriges Sekret und bedeckt Körper, Beine und Fühler mit Sand und Steinchen, sodaß sie äußerst schwer zu sehen ist.

48. Reduvius personatus L. Kam in Klausen ans Licht in 5 Expl.; lebt in Häusern.

49. Harpactor iracundus Poda. Häufig in Gardone und Klausen auf Büschen; im Sonnenschein gern fliegend.

50. - erythropus L. In Gardone häufiger als vorige, Larve und Imago namentlich unter Steinen; in Klausen nicht beobachtet.

\section{Capsidae.}

51. Miris laevigatus L. Riva, 1 Expl.

52. Megaloceraea longicornis Fab. Gardone, 2 Expl.

53. Calocoris chenopodii Fall. Gardone, häufig.

54. Camptobrochis lutescens Schill. Riva, 21. 5., 1 Expl.

55. Oncognathus binotatus F. Diese mehr aus dem nördlichen Mitteleuropa bekannte Art war in Gardone am Mte. Bartolomeo sehr häufig.

56. Capsus trifaciatus L. Klausen; 1 Expl. an einer Crataegushecke.

57. - laniarius L. Gardone; einzeln, aber häufig. 


\section{Hemiptera homoptera. \\ Cicadidae.}

1. Tettigia orni L. Von Mitte Juli an überall häufig auf Bäumen und an Telegraphenstangen.

2. Cicada plebeja Scop. $1 \sigma^{\top}$ an einem Kirschbaum, Klausen.

3. Tibicen haematodes Scop. Wie T. orni; hält sich besonders in Weinbergen auf.

4. Cicadetta tibialis $\mathrm{Pz}$. In Gardone sehr häufig an Berghalden auf Ginsterbüschen, die $\sigma^{\top} \mathrm{im}$ Sonnenschein zirpend, etwa ts .. ts .. ts .....; ist schon im Mai erwachsen.

\section{Fulgoridae.}

5. Cixius nervosus L. Riva, 20. 5., 1 ExpI.

6. - cunicularius L. Klausen, 1 Expl.

7. Issus coleoptratus Fab. Am Gardasee vereinzelt; springen sehr gewandt.

\section{Cercopidae.}

8. Triecphora vulnerata Ger. Sehr häufig an vielen Pflanzenarten, Gardaseegebiet.

9. - mactata Ger. Unter der vorigen, aber seltener.

10. Aphrophora salicis de Geer. Torbole, zahlreich auf Salix am Seeufer.

11. - alni Fall. Auf den feuchten Wiesen bei Sirmione sehr häufig.

12. Ptyelus spumarius L. Ebendaselbst.

\section{Membracidae.}

13. Centrotus cornutus L. Klausen; gern an Vincetoxicum. Jassidae.

14. Tettigonia viridis L. Wie A. alni.

\section{Erklärung zu Tafel II.}

Fig. 1. Blaps mucronata L. mit zweiteiliger Antenne (vergI. p. 12).

Fig. 2. Parnassius apollo forma decora Schultz.

Fig. 3. Chrysophanus alciphron gordius forma confluens Courv., trans.

Fig. 4. Lycaena amandus Schn.

Fig. 5. Callimorpha dominula forma insubrica Wack.

Fig. 6. Syntomis phegea forma aberr. 


\section{$2 \mathrm{BHL}$ Biodiversity Heritage Library}

Ramme, W. 1911. "Entomologische Ergebnisse einer Reise nach Oberitalien und Sudtirol." Berliner entomologische Zeitschrift / herausgegeben von dem Entomologischen Verein in Berlin 56, 11-32.

View This Item Online: https://www.biodiversitylibrary.org/item/36396

Permalink: https://www.biodiversitylibrary.org/partpdf/20084

\section{Holding Institution}

Smithsonian Libraries

\section{Sponsored by}

Smithsonian

\section{Copyright \& Reuse}

Copyright Status: NOT_IN_COPYRIGHT

This document was created from content at the Biodiversity Heritage Library, the world's largest open access digital library for biodiversity literature and archives. Visit BHL at https://www.biodiversitylibrary.org. 